\title{
RESEARCH
}

\section{Metabolomics signatures of a subset of RET variants according to their oncogenic risk level}

\author{
Charlotte Veyrat-Durebex 1,2, Nathalie Bouzamondo1, Morgane Le Mao², Juan Manuel Chao de la Barca1,2, \\ Céline Bris'1,2, Xavier Dieu1', Gilles Simard1', Cédric Gadras¹, Lydie Tessier¹, Delphine Drui³, Françoise Borson-Chazot4, \\ Anne Barlier5, Pascal Reynier ${ }^{1,2}$ and Delphine Prunier-Mirebeau, ${ }^{1,2}$ \\ 1Département de Biochimie et Génétique, CHU d'Angers, Angers, France \\ 2Equipe Mitolab, Institut MITOVASC, Unité Mixte de Recherche CNRS 6214, INSERM U1083, Université d'Angers, Angers, France \\ ${ }^{3}$ Service d'Endocrinologie, CHU de Nantes, Nantes, France \\ ${ }^{4}$ Hospices Civils de Lyon, Fédération d'Endocrinologie, Université Lyon 1, HESPER EA 7425, Lyon, France \\ ${ }_{5}^{5}$ Aix-Marseille University, CNRS, CRN2M, UMR 7286, and APHM La Conception Hospital, Molecular Biology Laboratory, Marseille, France
}

Correspondence should be addressed to C Veyrat-Durebex: charlotte.veyrat@live.fr

\begin{abstract}
Thirty percent of medullary thyroid carcinomas (MTCs) are related to dominant germline pathogenic variants in the RET proto-oncogene. According to their aggressiveness, these pathogenic variants are classified in three risk levels: 'moderate', 'high' and 'highest'. The present study compares the metabolomics profiles of five pathogenic variants, whether already classified or not. We have generated six stable murine fibroblast cell lines (NIH3T3) expressing the WT allele or variants of the human RET gene, with different levels of pathogenicity, including the M918V variant that is yet to be accurately classified. We carried out a targeted metabolomics study of the cell extracts with a QTRAP mass spectrometer, using the Biocrates Absolute IDQ p180 kit, which allows the quantification of 188 endogenous molecules. The data were then subjected to multivariate statistical analysis. One hundred seventy three metabolites were accurately measured. The metabolic profiles of the cells expressing the RET variants were found to be correlated with their oncogenic risk. In addition, the statistical model we constructed for predicting the oncogenic risk attributed a moderate risk to the M918V variant. Our results indicate that metabolomics may be useful for characterizing the pathogenicity of the RET gene variants and their levels of aggressiveness.
\end{abstract}

\section{Introduction}

Multiple endocrine neoplasia type 2 (MEN2) is a multiglandular tumor syndrome due to germline 'gain-offunction' variants in the REarranged during Transfection (RET) proto-oncogene (Mulligan et al. 1994). This gene encodes a tyrosine kinase transmembrane receptor required for the proliferation and differentiation of specific neural cells, such as the progenitors of the enteric nervous system, and neuroendocrine cells, especially thyroid C cells and adrenal chromaffin cells. Given its broad roles in tissue morphogenesis and proliferation, the RET gain-offunction mutations lead to MEN2 with medullary thyroid cancer (MTC), either alone or in combination with pheochromocytoma and primary hyperparathyroidism. Two distinct forms of this syndrome, MEN2A and MEN2B, are associated with distinct pathogenic variants in the RET gene (Wells et al. 2015).

Extensive study of the RET gene has led the American Thyroid Association (ATA) to recommend classification of 
each variant according to the occurrence and aggressiveness of MTC, to go beyond the distinction of pathogenic or not pathogenic. Three classes of risk have been defined: the 'highest' risk involving the RET variant M918T, the 'high' risk, including variants of RET codons 634 and A883F, and the 'moderate' risk, including all the other variants that have been sufficiently documented (Wells et al. 2015). Due to the excellent genotype-phenotype correlation, this classification of prognosis is essential for defining the age at which prophylactic thyroidectomy should be performed. It also allows the selection of patients that have to be screened for pheochromocytoma, hyperparathyroidism and other symptoms caused by these RET variants. In the MEN2 syndrome, RET mutations mostly occur in exons $5,8,10,11,13,14,15$ and 16 among the 20 exons composing the gene. Classically, MEN2A is associated with the substitution of a cysteine by another amino acid in the extracellular domain, and MEN2B, which presents a very specific phenotype, is associated with the M918T mutation in the intracellular tyrosine kinase domain.

In a recent communication, we evaluated the spectrum of RET mutations found in France between 2003 and 2013 (Lebeault et al. 2017). We found that $7.7 \%$ of the patients with MEN2 presented RET variants that were not classified by the ATA (Wells et al. 2015), a finding that compromised genetic counseling for patients and their families. To interpret the significance of these variants, we used guidelines based on various criteria such as population data, computational data, functional data and segregation data (Richards et al. 2015). These guidelines classify variants in five categories, that is, 'pathogenic', 'likely pathogenic', 'of uncertain significance', 'likely benign' and 'benign'. After this classification using the guidelines, 9 RET variants were classified as likely pathogenic, 6 as likely benign and 11 remained of uncertain significance. However, we were unable to categorize the potentially pathogenic RET variants unclassified by the ATA, in one of the three risk levels of the ATA recommendations based on the aggressiveness of MTC.

The mutation-related activation of the RET protein results in the major activation of cell proliferation signaling pathways, including the MAPK/ERK, PI3K/AKT, nuclear factor $\kappa \mathrm{B}(\mathrm{NF \kappa B}), \mathrm{JAK} / \mathrm{STAT}$ and JNK pathways (Mulligan 2014). The activation of oncogenes is frequently associated with metabolic reprogramming. The common mutation V600E in the BRAF oncogene, found in melanoma and other cancers, induces increased mitochondrial respiration and redox stress by activating the mitochondrial pyruvate dehydrogenase enzyme (Kaplon et al. 2013). Yun et al. have shown that cells expressing the BRAF or KRAS mutations raise the glycolytic rate by increasing the glucose uptake through overexpression of the glucose transporter GLUT1, as well as by increasing the production of lactate (Yun et al. 2009).

Functional studies aimed at determining the pathogenicity of RET variants are usually based on the ability of transfected variants to activate cell transformation and oncogenic signaling pathways. In the present study, we investigated the possibility of metabolomics signatures contributing to the classification of RET mutations according to their pathogenicity. We applied a quantitative targeted metabolomics approach to analyze the lipids, sugars, carnitines, amino acids and biogenic amines, in combination with assessment of the mitochondrial function, in cells expressing the WT or mutated RET protein. We then used the metabolomics signature observed at various levels of oncogenic risk to predict the risk level of the M918V variant, unclassified by the ATA. We chose to study the variant of uncertain significance (VUS) M918V since a functional study of this variant had been previously performed (Cosci et al. 2011), and this variant seems to have a moderate or no oncogenic level despite the fact that it occurs in the residue known to harbor the highest pathogenic mutation (M918T).

\section{Materials and methods}

\section{Construction of the RET mutants}

All RET mutants were obtained by site-directed mutagenesis of the RET9 WT plasmid (p-Babe-RET9 vector, kindly provided by Dr Massimo Santoro, Napoli, Italy) using an in vitro mutagenesis system (Quik-Change site-directed mutagenesis, Agilent). Five missense mutations were introduced into the RET9 plasmid: two variants associated with a 'moderate' oncogenic risk, that is, L790F and V804M; a 'high'-risk variant, that is, C634R; the 'highest' risk variant, that is, M918T; and a variant of unknown significance (VUS), that is, M918V. The WT allele was used as the control. Table 1 shows details of the variants.

\section{Cell culture and transfection}

The NIH3T3 cell line, obtained from the American Type Culture Collection (ATCC), was maintained in DMEM with F12 nutrient (DMEM-F12, PAN BIOTECH, Aidenbach, Germany) supplemented with 1\% L-glutamine, 1\% penicillin-streptomycin and $10 \%$ fetal bovine serum (FBS, PAN BIOTECH). Transfection was performed using Lipofectamine 2000 (Invitrogen), and the selection 
Table 1 Description of the RET variants (transcript NCBI NM_020630.4).

\begin{tabular}{|c|c|c|c|}
\hline ATA classification & cDNA variant & Protein variant & $\begin{array}{l}\text { Short name } \\
\text { of variant }\end{array}$ \\
\hline Moderate & c. $2370 G>T$ & p.Leu790Phe & L790F \\
\hline Moderate & c. $2410 \mathrm{G}>\mathrm{A}$ & p.Val804Met & V804M \\
\hline High & c.1900T>C & p.Cys634Arg & C634R \\
\hline Highest & c. $2753 \mathrm{~T}>\mathrm{C}$ & p.Met918Thr & M918T \\
\hline Non-classified (VUS) & c. $2752 A>G$ & p.Met918Val & M918V \\
\hline
\end{tabular}

of NIH3T3 stable clones was done using puromycin (Invitrogen).

The growth of each cell line stably transfected with the WT or mutant RET gene was assessed. The different stable cell lines were plated in six-well plates and treated with $100 \mathrm{ng} / \mu \mathrm{L}$ of mouse glial cell-derived neurotrophic factor (GDNF) (SRP3200, Sigma-Aldrich) during 72 h. Cell counts were made on six replicates.

\section{Real-time PCR}

Cell DNA was extracted using the EZ1 DNA extraction automate (Qiagen). Real-time PCR was performed using iQ SYBR Green Supermix (Bio-Rad) and specific primers for the RET gene, on a CFX96 Touch Real-Time PCR Detection System (Bio-Rad), according to the manufacturer's instructions.

\section{Western blot}

Cells were lysed in water with protease inhibitors. The proteins, separated by SDS-PAGE in a $4-15 \%$ polyacrylamide gel (Bio-Rad), were transferred onto a PVDF membrane using the Trans-Blot Turbo system (Bio-Rad). Protein blots were probed with various primary antibodies from Cell Signaling for RET (14556S), AKT (9272S), p-AKT (13038S), 44/42 MAPK (ERK) (4695S), p-44/42 MAPK (p-ERK) (9101S) and from Abcam for $\alpha$-tubulin (ab7291), diluted to $1 / 1000$, followed by horseradish peroxidase-conjugated anti-rabbit or anti-mouse secondary antibodies, diluted to $1 / 10,000$ and revealed by an enhanced chemiluminescence substrate (Pierce-Thermo Fischer Scientific), according to the manufacturer's instructions.

\section{Assessment of mitochondrial respiration}

The oxygen consumption rate (OCR) was measured in intact, adherent fibroblasts with an XF96 Extracellular Flux Analyzer (Seahorse Bioscience, Billerica, MA, USA). Cells were seeded in Seahorse XF 96-well cell culture microplates (Seahorse Bioscience) at a density of $2 \times 10^{4}$ cells/well in $100 \mu \mathrm{L}$ of culture medium and incubated for $4 \mathrm{~h}$ at $37^{\circ} \mathrm{C}$ in an atmosphere of $5 \% \mathrm{CO}_{2}$. After replacing the growth medium with $180 \mu \mathrm{L}$ of bicarbonate-free DMEM pre-warmed to $37^{\circ} \mathrm{C}$, the cells were pre-incubated for $1 \mathrm{~h}$ at $37^{\circ} \mathrm{C}$ without $\mathrm{CO}_{2}$ before starting the experiment. The optimal concentration of carbonyl cyanide 4-(trifluoromethoxy)-phenylhydrazone (FCCP) required for maximal stimulation of respiration, which ranged between 2 and $14 \mu \mathrm{M}$, was routinely determined by titration. After determining the basal OCR of the cells, oligomycin $(1 \mu \mathrm{M})$, FCCP $(2-14 \mu \mathrm{M})$ and antimycin A ( $2 \mu \mathrm{g} / \mathrm{mL}$ ), were sequentially added to the media and the OCR was quantified. The data were expressed as pmol of $\mathrm{O}_{2}$ per minute, normalized by cell number.

\section{Targeted metabolomics analysis}

For metabolomics analysis, NIH3T3 cell lines were maintained in medium as described above without GDNF supplementation. When the cell culture achieved a confluence of 70-80\%, adherent cells were washed with phosphate-buffered saline (PBS). Cells were then harvested using trypsin, washed and centrifuged $(300 \boldsymbol{g}$ for $5 \mathrm{~min}$ at $4^{\circ} \mathrm{C}$ ). Metabolic quenching was performed by adding $100 \mu \mathrm{L}$ of a solution of cold methanol/PBS $(85: 15, \mathrm{v} / \mathrm{v})$ to the cell pellet (approximately $3 \times 10^{6}$ cells). The mixture was transferred to a $0.5 \mathrm{~mL}$ homogenizer tube prefilled with $1.4 \mathrm{~mm}$ ceramic beads (Bertin Instruments, Montigny-leBretonneux, France). Cell lysis and metabolite extraction was achieved in a Precellys 24 homogenizer (Bertin Instruments) by two cycles of grinding (40 s at $6500 \mathrm{rpm}$, followed by $30 \mathrm{~s}$ at $6000 \mathrm{rpm}$ ) at $4^{\circ} \mathrm{C}$. The resulting homogenate was centrifuged at $10,000 \mathrm{~g}$ for $5 \mathrm{~min}$ at $4^{\circ} \mathrm{C}$ and the supernatant was stored at $-80^{\circ} \mathrm{C}$ until analysis. Ten replicates of each stable cell line were performed with a difference of at most two cell passages between the ten samples.

We applied a targeted quantitative metabolomic approach to cell extracts using the Biocrates AbsoluteIDQ p180 kit (Biocrates Life sciences, Innsbruck, Austria). This kit, in combination with an AB Sciex QTRAP 5500 mass spectrometer (Life Sciences SCIEX, Framingham, MA, USA), allows the quantification of 188 different endogenous molecules, including acylcarnitines, amino acids, biogenic amines, glycerophospholipids, sphingolipids and sugars. After thawing on ice, $10 \mu \mathrm{L}$ of each sample (supernatants of the cell-lysate homogenate) were prepared according to the Biocrates Kit User Manual with ready-to-use solutions. Flow injection analysis coupled with tandem mass spectrometry (FIA-MS/MS) (c) 2019 Society for Endocrinology Published by Bioscientifica Ltd. Printed in Great Britain 
was used for the analysis of carnitine, acylcarnitines, lipids and hexoses. Liquid chromatography (LC) was used for separating amino acids and biogenic amines before quantitation with mass spectrometry. After the validation of quality controls, the concentrations of metabolites were normalized to concentrations of protein cell extracts.

\section{Statistical analysis}

Multivariate data analyses were performed using the Simca P+ version 14.0 software (Umetrics, Umea, Sweden). Data were log-transformed and unit-variance scaled. We first performed unsupervised Principal Component Analysis (PCA) and so identified the similarity or the differences between sample metabolic profiles. We then used supervised analysis, the Orthogonal Partial Least Squares - Discriminant Analysis (OPLS-DA), to classify samples. OPLS-DA evaluated variations between groups for each metabolite (partitioning variations that correlated with the class identifier and variations that did not contribute to discrimination between groups). The result was an OPLS-DA built from one 'predictive' component and two or more orthogonal components. The significance of the OPLS-DA models was assessed using the CV-ANOVA test, the $\mathrm{Q}^{2}$ Ycum (goodness of prediction) and the $\mathrm{R}^{2}$ Ycum (goodness of fit) values. The most relevant variables were determined from the Variable Importance for the Projection (VIP). VIP values represent the importance of these variables for the OPLS-DA models between the $Y$ and $X$ variables. Score plots represent the $2 \mathrm{D}$ projection of the two main components of models allowing the distribution and grouping of the samples, each dot representing a sample, each color a group of samples.

Univariate analysis was performed using MetaboAnalyst 3.0 (www.metaboanalyst.ca/). Mean comparisons of metabolite concentrations were performed using the non-parametric Wilcoxon test, and the Benjamini-Hochberg correction was applied to multiple tests. The results of the univariate analysis were summarized in a $2 \mathrm{D}$ graph known as the volcano plot with between-group metabolite fold change values on the $x$-axis and $\log$-scaled $P$ values on the $y$-axis.

\section{Analysis of metabolic pathways}

For the biological interpretation of the metabolite dataset, we mapped the quantified metabolites to the KEGG pathway database (Kyoto Encyclopedia of Genes and Genomes; www.genome.jp/kegg/), using MetaboAnalyst 3.0, a comprehensive online tool suite for metabolomics data analysis and interpretation. This analysis was performed using the discriminant metabolites, excluding phosphatidylcholines (PCs) since their characterization cannot differentiate between the fatty acid chains.

\section{Results}

\section{Characterization of the in vitro model}

The copy number of the integrated RET gene was checked in the different NIH3T3 clones by real-time PCR to select cell lines possessing an equivalent copy number of the RET gene. The expression of the RET protein was verified by Western blotting (Supplementary Fig. 1, see section on supplementary data given at the end of this article).

The morphology of cell lines over-expressing the mutated RET appeared to be modified, especially in cells carrying the RET-M918T mutation, which appeared particularly smaller (Fig. 1A). We have explored MAPK/ERK signaling and AKT signaling using Western blot. As expected, we highlighted higher activation of ERK and AKT signaling, through higher phosphorylation of these proteins, in cells expressing M918T RET variant (Fig. 1B). Cells expressing C634R and L790F RET mutations also showed higher phosphorylation of ERK and AKT proteins. WT or other RET mutations did not present relevant differences for phosphorylation of both ERK and AKT. After $72 \mathrm{~h}$ of treatment with the GDNF, cell growth rates were significantly higher in all RET-mutant cells compared to RET-WT cells $(P=0.0021$ for the five mutant cells) (Fig. 1C). The rate of cell proliferation was not statistically different between the mutants, except for RET-M918T cells, which have a higher growth rate compared to other RET mutant cells $(P=0.0002)$ (Fig. $1 C$ ).

\section{Cell lines overexpressing the human RET receptor showed no significant variation of mitochondrial respiration}

We determined the mitochondrial respiratory rate as the mean of the cellular oxygen consumption in the different cell lines stably transfected with the RET gene. The basal and maximal cellular OCRs did not differ from one cell line to the other in all the cell lines studied. Mitochondrial complex III was inhibited by antimycin in order to evaluate the non-mitochondrial respiration. We observed no difference between the cell lines studied in terms of basal respiration, phosphorylating and nonphosphorylating respiration and the capacity of the electron transfer system (Supplementary Table 1). (c) 2019 Society for Endocrinology Published by Bioscientifica Ltd. Printed in Great Britain 
B
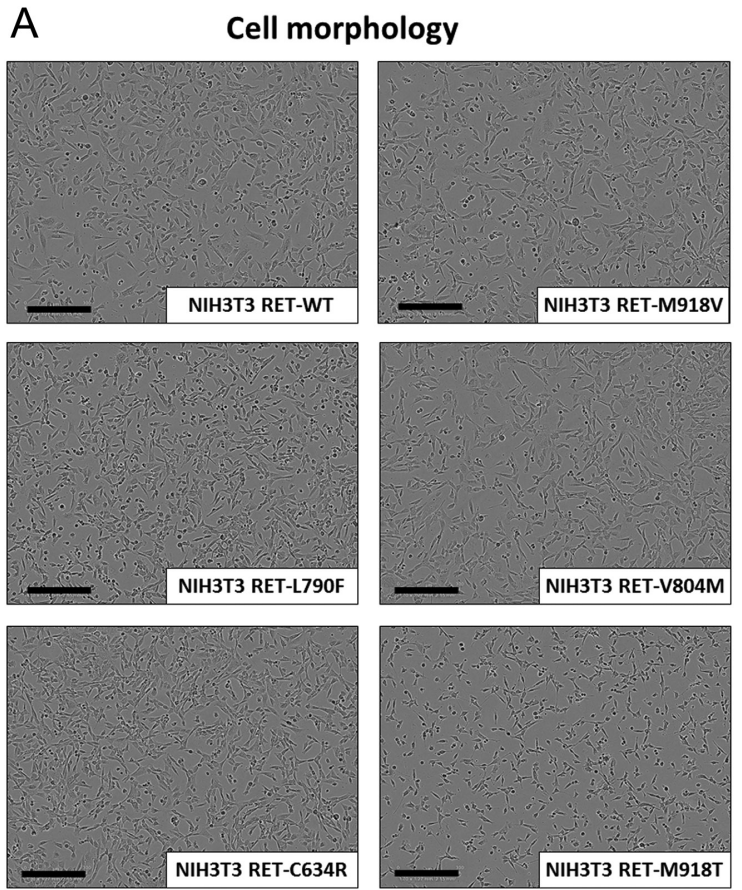

C
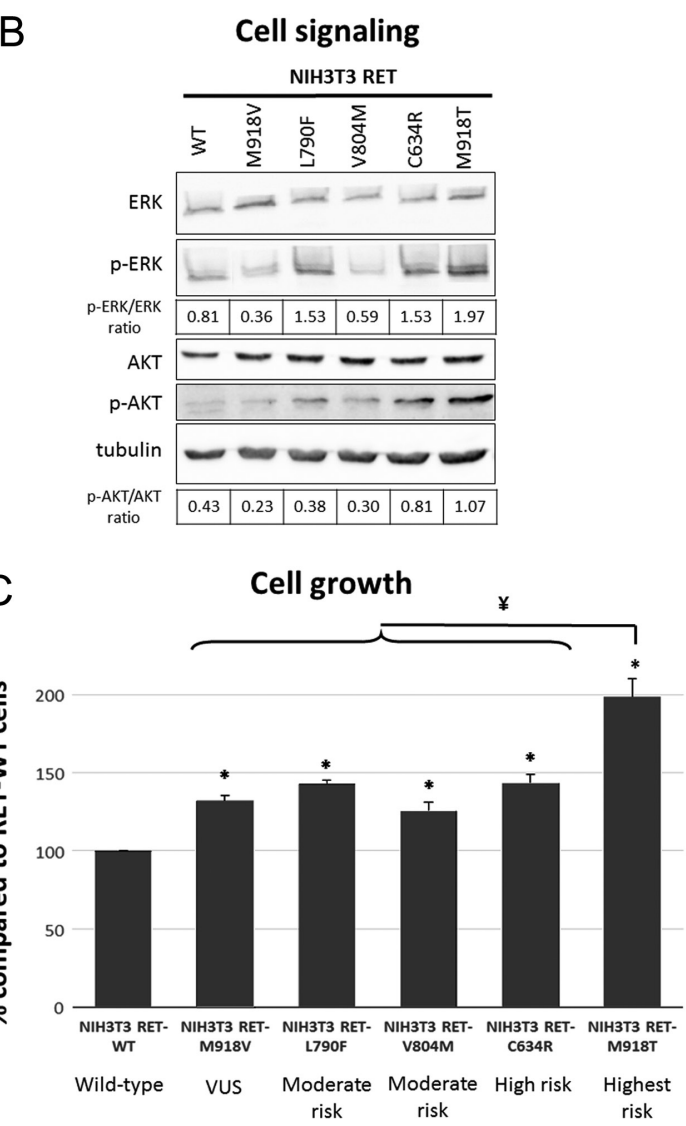

\section{Figure 1}

(A) Cell morphology. Pictures of the cell cultures stably transfected with RET WT or mutant (C634R, L790F, V804M, M918V and M918T). Scale bars represent $300 \mu \mathrm{m}$. (B) Cell signaling. Western blots of ERK, p-ERK, AKT and p-AKT proteins in cell lines expressing mutant or WT RET and the ratio of phosphorylated protein related to non-phosphorylated were calculated. (C) Cell growth. The cell growth (percentage compared to RET-WT NIH3T3 cells considered as 100\%) of cell cultures stably transfected with RET mutants (C634R, L790F, V804M, M918V and M918T), after 72 h of GDNF treatment. All RET mutant cells had a significantly higher growth rate compared to WT cells ( $* P<0.01$ ); and RET-M918T cells had a higher growth rate compared to all the other mutant cells $(\because P=0.0002)$.

\section{Cells expressing human RET have distinct metabolomics signatures}

Among the 188 metabolites analyzed by the Biocrates p180 kit, 173 were accurately measured in the NIH3T3 cells (Supplementary Table 2).

Applying non-supervised multivariate analysis by PCA to the six transfected cell lines and the non-transfected cell line, we highlighted a spontaneous clustering of the non-transfected NIH3T3 cell samples (NIH3T3 NT), the RET-M918T NIH3T3 and the RET-WT NIH3T3 (Fig. 2A). The other cell lines with pathogenic RET mutants (RET-C634R, RET-L790F, RET-V804M) and the VUS RET-M918V were not distinguished.

Discriminant analysis of these seven cell lines, using supervised multivariate analysis OPLS-DA, distinguished between the specific metabolomic signatures of the cell lines, with four clusters (Fig. 2B). Non-transfected (NIH3T3
NT) cells and RET-WT cells formed two distinct clusters, highlighting the strong influence of RET expression on the cell metabolomics profiles. Moreover, the model distinguished a specific metabolomics profile for mutated RET cells (RET-C634R, RET-L790F, RET-V804M and RET-M918V), which formed a cluster at the center of the score plot (Fig. 2B). RET-M918T cell samples formed a separate group, distinct form the other RET mutated cell lines, highlighting the specific metabolomics signature of these cells, which harbored the 'highest' risk RET mutation causing the MEN2B syndrome (Fig. 2B).

Excluding the non-transfected cells, another OPLS-DA model also showed the clustering of cells expressing the RET mutant and RET-WT cells (Fig. 3A). Univariate analysis of metabolomics data discriminated between these two groups. The volcano plot (i.e. fold change $>2$ and $P$ value $<0.05$ ), showed a significant decrease of unsaturated, very long-chain phosphatidylcholine 

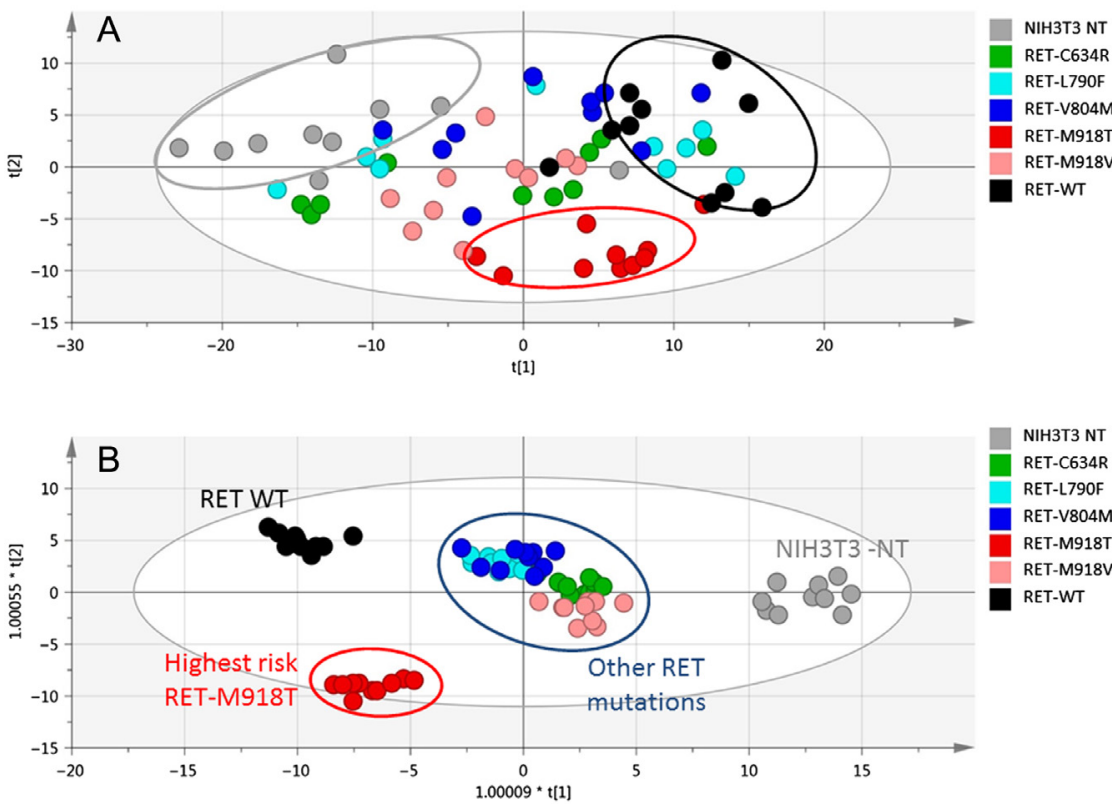

Figure 2

Cells expressing human RET have distinct metabolomics signatures. (A) PCA plot obtained from metabolomics profiles of all the samples studied: non-transfected NIH3T3 cells (NIH3T3-NT) (grey dots), transfected with RET-WT (black dots), or mutant RET (C634R green dots, L790F light blue dots, V804M blue dots, M918T red dots and $\mathrm{M} 918 \mathrm{~V}$ light red dots). $R^{2} X=0.841$ and $Q^{2}=0.796$. (B) OPLS-DA plot of the model performed according to the seven cell types (non-transfected or transfected with RET plasmids). Non-transfected NIH3T3 cells (NIH3T3-NT) (grey dots); cells transfected with RET-WT (black dots), or mutant RET (C634R green dots, L790F light blue dots, V804M blue dots, $\mathrm{M} 918 \mathrm{~T}$ red dots and $\mathrm{M} 918 \mathrm{~V}$ light red dots). $R^{2} X=0.89, R^{2} Y=0.849$ and $Q^{2}=0.616$. A full color version of this figure is available at https://doi. org/10.1530/ERC-18-0314.
(PC) species in mutant RET cells compared to RET-WT cells (Fig. 3B).

\section{The metabolomics profiles of cells expressing mutated human RET differed according to the oncogenic risk levels}

Supervised OPLS-DA analysis carried out according to risk level, with RET-L790F and RET-V804M carrying a 'moderate' risk, RET-C634R a 'high' risk and RET-M918T the 'highest' risk, revealed a discriminant model (Fig. 4A). Using the predictive variation between $X$ (metabolites) and $Y$ (cells samples) variables given by $R^{2} X$ (cum), the model interpreted approximately $87.9 \%$ of the total variation in $X$. This model explained approximately $95.8 \%$ of the variations in the $Y$ vector $\left(R^{2} Y(\mathrm{cum})\right)$. The predictive value of the model was considered good with a $Q^{2}$ (cum) of 0.87 . The value of the CV-ANOVA test $\left(P=1.92 \times 10^{-12}\right)$ excluded the risk of over-fitting. The analysis highlighted a particularly high specific metabolic signature for the

\section{A Multivariate analysis}

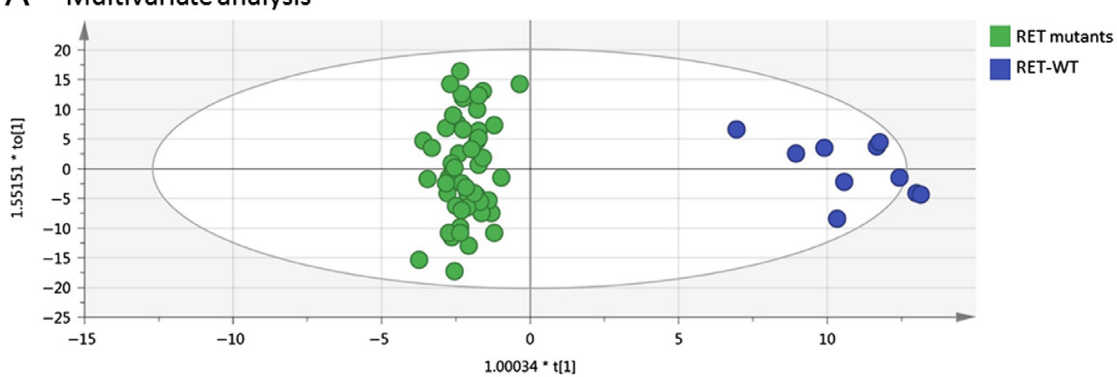

B Univariate analysis

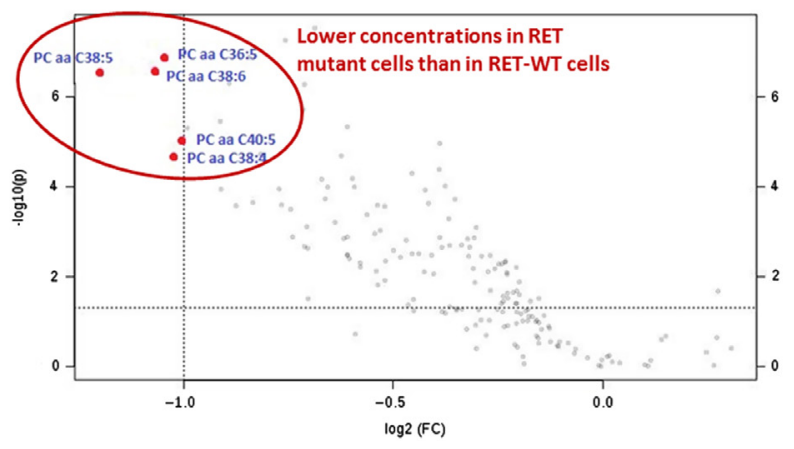

Figure 3

Cells expressing mutant or WT RET have distinct metabolic profiles. (A) The OPLS-DA plot based on cells overexpressed mutant or WT RET. NIH3T3 cells transfected with WT RET (blue dots) or with mutant RET (green dots). $R^{2} X=0.819, R^{2} Y=0.963$ and $Q^{2}=0.839$. (B) Volcano plot of the univariate analysis of the metabolomics data of the two groups (WT and mutant RET), using fold change $>2$ and $P$-value $<0.05$ as thresholds. Graph was built with between-group metabolite fold change values on the $x$-axis, and log-scaled $P$-values on the $y$-axis. A full color version of this figure is available at https://doi.org/10.1530/ERC-18-0314 


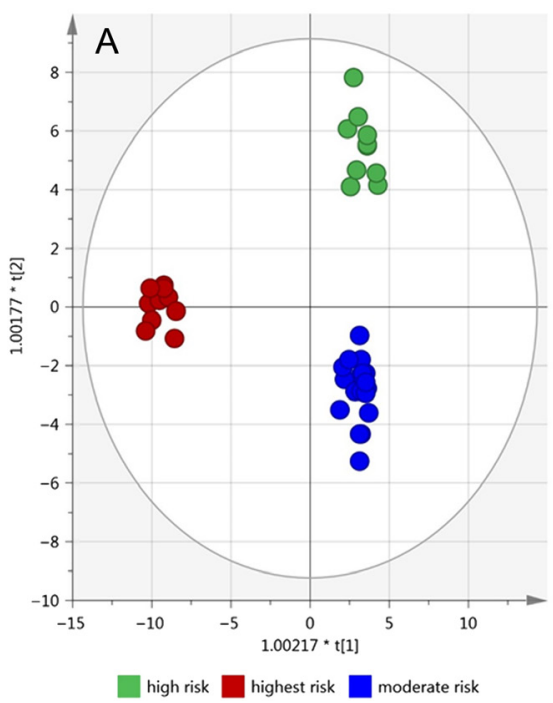

B

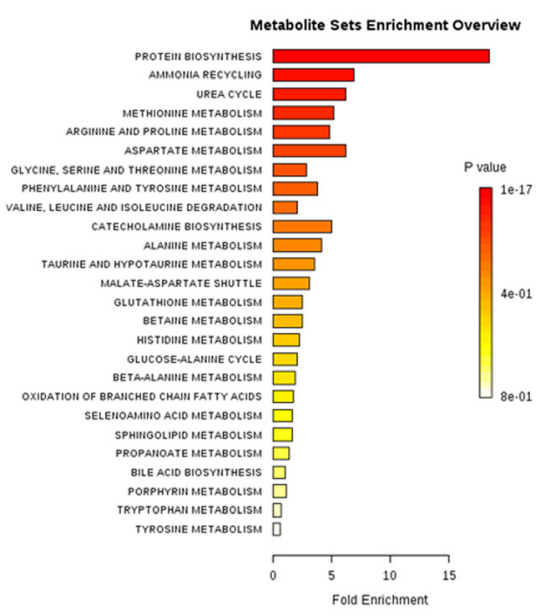

Figure 4

The metabolomics profiles of cells expressing mutated human RET differ according to the risk level. (A) Score plot of the OPLS-DA model performed according to the level of oncogenic risk of RET mutations and obtained from the metabolomics profiles of NIH3T3 cells transfected with various mutants of RET: C634R as a 'high' risk mutant (green dots), L790F and V804M as 'moderate' risk mutants (blue dots), and M918T as the 'highest risk' mutant (red dots). $R^{2} X=0.879$, $R^{2} Y=0.958$, and $Q^{2}=0.87$. (B) Metabolic pathway analysis of discriminant metabolites according to the risk level of RET mutations. Metabolic pathway analyses related to the metabolites that were discriminant in the OPLS-DA of the level of oncogenic risk of RET mutations in cell lines using the MetaboAnalyst tools. The figure graphically summarizes the analysis of metabolite set enrichment for discriminant metabolites. The horizontal bars summarize the main metabolite sets identified in this analysis; the bars are colored according to their $P$-values, and the length is based on the fold enrichment. A full color version of this figure is available at https://doi. org/10.1530/ERC-18-0314. highest risk RET-M918T mutated cells. The 59 metabolites supporting this discriminant model (metabolites with a VIP score $>1$ ) are shown in Table 2. Among the 59 discriminant metabolites, 19 were amino acids with relatively lower concentrations in the RET-M918T cells with the 'highest' risk compared to 'high'- and 'moderate'risk cells. These differences were found significant using univariate analysis $(P<0.0001)$. Moreover, 24 of the 59 discriminant metabolites were acylcarnitines, 14 of which showed, by univariate analysis, significant differences in the 'highest' risk cells compared to 'high' and 'moderate' risk cells (Table 2). Levels of long-chain acylcarnitines were relatively lower in RET-M918T cells, whereas levels of short-chain acylcarnitines were relatively higher in this group.

Figure $4 \mathrm{~B}$ shows the results of pathway analysis using the most discriminant metabolites between cells with the RET mutation with the 'highest' risk and those with 'high' or 'moderate' risks. Protein biosynthesis, the recycling of ammonia, the urea cycle and the metabolism of various amino acids were the most enriched pathways.

\section{The risk level of RET mutation can be predicted using the metabolomics profile}

We tested the prediction of the risk level for the VUS RET-M918V, using the OPLS-DA model generated with various RET mutations having a well-defined classification. A new dataset including the data of RET-M918V cells was inserted in the OPLS-DA model and analyzed using the prediction tools of SIMCA software (Fig. 5). Ninety percent of RET-M918V cells were predicted as probably at moderate risk. The prediction of the model failed for only one of the ten RET-M918V samples examined.

\section{Discussion}

Metabolic alterations are considered to be emerging hallmarks of cancer as well as possible therapeutic targets (Hanahan \& Weinberg 2011). We used a massspectrometry-based, targeted quantitative approach allowing quantification of 173 intracellular metabolites on a well-defined in vitro model expressing the WT or mutant RET oncogene. We highlighted phosphatidylcholines (PC) as a group of molecules differentially regulated between RET-WT and RET mutant cells. We also showed that the RET-M918T mutant cells carrying the 'highest' oncogenic risk had a specific metabolic profile characterized by highly disturbed amino acid and acyl-carnitine metabolisms.

The activation of oncogenes has been associated with the deregulation of metabolism and the proliferative capacity of cells. Indeed, in our study, RET-WT cells had a lower growth rate and a distinct metabolic signature compared to cells expressing the RET oncogene with activating mutations. Tumor growth leads to an increased turnover of membrane lipids, which play an important role in signaling and metabolic pathways. 
Table 2 Metabolites with VIP scores $>1$ in the OPLS-DA model based on risk level groups and results of the univariate comparison (non-parametric Wilcoxon test and correction for multiple tests) of their concentrations between RET-M918T cells and other RET mutant cells.

\begin{tabular}{|c|c|c|c|}
\hline \multirow[b]{2}{*}{ VIP metabolites } & \multirow{2}{*}{$\begin{array}{c}\text { Multivariate analysis } \\
\text { VIP score }\end{array}$} & \multicolumn{2}{|c|}{ Univariate analysis } \\
\hline & & Change in M918T vs other mutants & $P$ value \\
\hline Propionylcarnitine & 1.6701 & $\downarrow$ & 0.0134 \\
\hline LysoPC a C20:4 & 1.4890 & $=$ & 0.0689 \\
\hline Taurine & 1.4256 & $\downarrow$ & $<0.0001$ \\
\hline Octadecenoylcarnitine & 1.4149 & $\downarrow$ & 0.0001 \\
\hline Alanine & 1.4004 & $\downarrow$ & $<0.0001$ \\
\hline Tyrosine & 1.3885 & $\downarrow$ & $<0.0001$ \\
\hline Phenylalanine & 1.3844 & $\downarrow$ & $<0.0001$ \\
\hline Isoleucine & 1.3835 & $\downarrow$ & $<0.0001$ \\
\hline Hexadecanoylcarnitine & 1.3734 & $\downarrow$ & 0.0001 \\
\hline Arginine & 1.3636 & $\downarrow$ & 0.0002 \\
\hline Proline & 1.3608 & $\downarrow$ & $<0.0001$ \\
\hline Leucine & 1.3596 & $\downarrow$ & $<0.0001$ \\
\hline Threonine & 1.3592 & $\downarrow$ & $<0.0001$ \\
\hline Glycine & 1.3580 & $\downarrow$ & $<0.0001$ \\
\hline Trans-4-hydroxyproline & 1.3570 & $\downarrow$ & $<0.0001$ \\
\hline Asparagine & 1.3363 & $\downarrow$ & $<0.0001$ \\
\hline Hexadecenoylcarnitine & 1.3157 & $\downarrow$ & 0.0029 \\
\hline Methionine & 1.3133 & $\downarrow$ & $<0.0001$ \\
\hline Aspartate & 1.3109 & $\downarrow$ & $<0.0001$ \\
\hline Glutamate & 1.3067 & $\downarrow$ & $<0.0001$ \\
\hline Valine & 1.3044 & $\downarrow$ & $<0.0001$ \\
\hline Tryptophan & 1.2931 & $\downarrow$ & $<0.0001$ \\
\hline Hydroxyoctadecenoylcarnitine & 1.2827 & $=$ & 0.2444 \\
\hline Histidine & 1.2824 & $\downarrow$ & $<0.0001$ \\
\hline Ornithine & 1.2574 & $\downarrow$ & 0.0001 \\
\hline Hydroxybutyrylcarnitine & 1.2068 & $\uparrow$ & 0.0071 \\
\hline Methionine sulfoxide & 1.2005 & $\downarrow$ & $<0.0001$ \\
\hline Spermidine & 1.1987 & $=$ & 0.6801 \\
\hline Butyrylcarnitine & 1.1953 & $=$ & 0.2072 \\
\hline LysoPC a C14:0 & 1.1900 & $\uparrow$ & 0.0014 \\
\hline Serine & 1.1724 & $\downarrow$ & $<0.0001$ \\
\hline Citrulline & 1.1696 & $=$ & 0.4375 \\
\hline Hydroxyhexadecenoylcarnitine & 1.1694 & $=$ & 0.7525 \\
\hline LysoPC a C18:2 & 1.1569 & $=$ & 0.7342 \\
\hline LysoPC a C17:0 & 1.1535 & $\downarrow$ & 0.0727 \\
\hline Propenoylcarnitine & 1.1332 & $\uparrow$ & 0.0082 \\
\hline Octadecadienylcarnitine & 1.1315 & $=$ & 0.4818 \\
\hline LysoPC a C20:3 & 1.1276 & $=$ & 0.7710 \\
\hline Hydroxypropionylcarnitine & 1.1028 & $\uparrow$ & 0.0036 \\
\hline Octadecanoylcarnitine & 1.1027 & $\downarrow$ & 0.0095 \\
\hline alpha-Aminoadipic acid & 1.1024 & $\downarrow$ & 0.0001 \\
\hline Butenylcarnitine & 1.1016 & $\uparrow$ & 0.0117 \\
\hline PC ae C30:1 & 1.0883 & $\uparrow$ & 0.0001 \\
\hline Glutarylcarnitine (hydroxyhexanoylcarnitine) & 1.0785 & $\uparrow$ & 0.0011 \\
\hline Tiglylcarnitine & 1.0702 & $\uparrow$ & 0.0349 \\
\hline Hexanoylcarnitine (fumarylcarnitine) & 1.0660 & $\uparrow$ & 0.0071 \\
\hline PC aa C26:0 & 1.0623 & $\uparrow$ & 0.0018 \\
\hline Dodecenoylcarnitine & 1.0546 & $=$ & 0.2444 \\
\hline Lyso PC a C28:0 & 1.0497 & $\uparrow$ & $<0.0001$ \\
\hline Tetradecenoylcarnitine & 1.0485 & $=$ & 0.0991 \\
\hline Hydroxyhexadecanoylcarnitine & 1.0464 & $=$ & 0.3442 \\
\hline Dodecanoylcarnitine & 1.0455 & $\uparrow$ & 0.0257 \\
\hline Methylglutarylcarnitine & 1.0397 & $\uparrow$ & 0.0076 \\
\hline
\end{tabular}


Table 2 Continued.

\begin{tabular}{l} 
viP metabolites \\
\hline Hexadecadieylcarnitine \\
PC ae C36:0 \\
Pimelylcarnitine \\
PC ae C32:1 \\
Valerylcarnitine \\
LysoPC a C24:0
\end{tabular}

\begin{tabular}{c} 
Multivariate analysis \\
\hline VIP score \\
\hline 1.0375 \\
1.0670 \\
1.0305 \\
1.0203 \\
1.0068 \\
1.0035 \\
\hline
\end{tabular}

\begin{tabular}{crr}
\hline \multicolumn{2}{c}{ Univariate analysis } & \\
\hline \multicolumn{1}{c}{ Change in M918T vs other mutants } & & $P$ value \\
\cline { 1 - 1 }$&{ } &{0.0991} \\
{\uparrow} &{\mathbf{0 . 0 0 3 9}} \\
{\uparrow} &{\mathbf{0 . 0 0 2 1}} \\
{=} &{\mathbf{0 . 0 0 0 7}} \\
{\uparrow} &{0.1265} \\
{ } &{<\mathbf{0 . 0 0 0 1}} \\
{\hline}$
\end{tabular}

Bold $P$ values indicate statistical significance.

We highlighted a decrease of levels of unsaturated, very long-chain PC species in RET mutant cells compared to RET-WT cells that could be explained by an excess of catabolism or by a synthesis defect. PCs are cleaved by a specific phospholipase $\mathrm{C}$, generating diacylglycerol and phosphocholine into cytosol. In fact, increased activity of the PC-specific phospholipase $\mathrm{C}$ enzyme has been reported in many cancers (Glunde et al. 2011, Cheng et al. 2016). Moreover, PCs might also be an important source of second messengers capable of acting on the MAPK/ERK and PI3K/AKT signaling pathways (Glunde et al. 2011, Gandola et al. 2014). These hypotheses may explain the lipidomic profile observed in this study.

Supervised multivariate analysis also demonstrated a robust separation between the different RET mutants according to their oncogenic risk. Pathway enrichment analysis, using the deregulated metabolites involved in the discrimination of the three classes of RET mutations, highlighted a sharp deregulation of the protein biosynthesis, the recycling of ammonia, and the urea cycle. Cells expressing the RET-M918T mutation, that is, the mutation with the highest level of risk, showed further metabolic adaptations, probably to support an increased cell proliferation rate, increasing the consumption of many amino acids with significantly reduced intracellular concentrations. Moreover, RET-M918T cells had significant variations in the levels of acylcarnitines compared to other mutant cells. Since acylcarnitines are energetic substrates for fatty acid oxidation (Reuter \& Evans 2012), these variations may be due to increased mitochondrial fatty acid beta-oxidation, supporting the increased energy demands of cell growth and proliferation (Brunelli et al. 2016). The stable mitochondrial respiration in the presence of RET mutants observed in our study is consistent with this hypothesis. Deep metabolic disorders observed in RET-M918T cells are consistent with the very specific phenotype observed in MEN2B and reinforce the importance of distinguishing MEN2B from MEN2A.

Using the multivariate discriminant model of the three classes of risk levels, we built a predictive model for the classification of the RET-M918V mutant that predicted $90 \%$ of the RET-M918V cell lines as carrying a moderate risk of aggressive cancer. Moreover, the study of the proliferation rate of these cells highlighted a growth pattern similar to that found in cells associated with a moderate risk. These results are consistent with functional studies advocating the classification of RET-M918V VUS as an RET mutation carrying a moderate risk (Cosci et al. 2011). Moreover, a recent clinical study on eight patients with MTC alone and lacking the MEN2B phenotype,

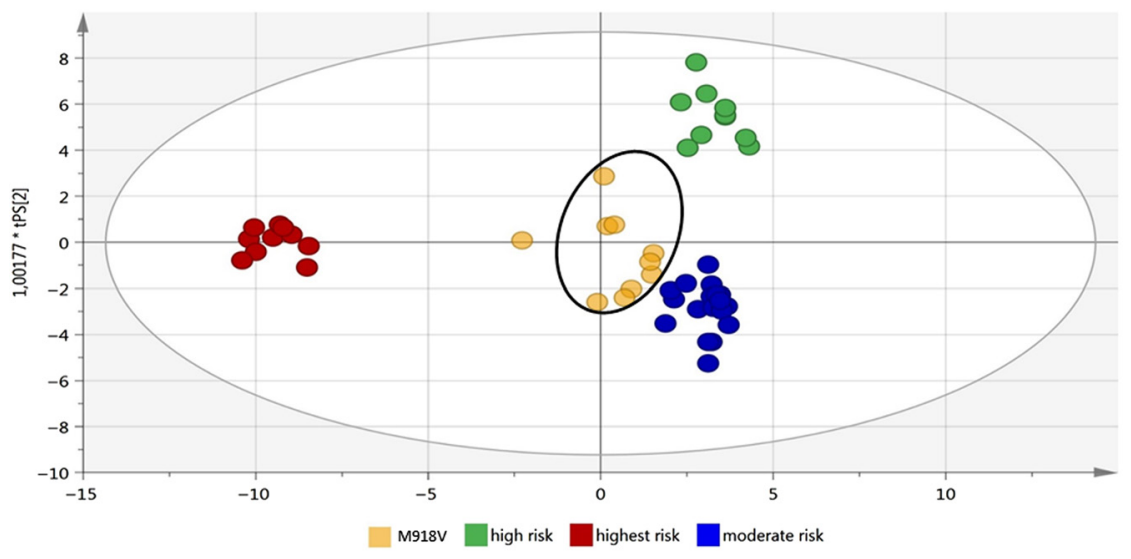

Figure 5

Prediction of the risk level of NIH3T3 RET-M918V cells. Score plot of the prediction of the risk level of NIH3T3 RET-M918V cells using the OPLS-DA model for the level of oncogenic risk of RET mutations obtained from the metabolomics profiles of NIH3T3 cells transfected with various mutants of RET (Fig. 4A): C634R as a 'high' risk mutant (green dots), L790F and V804M as 'moderate' risk mutants (blue dots), M918T as the 'highest risk' mutant (red dots), and M918V as unclassified mutant (light orange dots). $90 \%$ of RET-M918V cells are predicted to be at moderate risk (black circle). A full color version of this figure is available at https://doi.org/10.1530/ERC-180314. 
also suggested that M918V should be considered as carrying a moderate risk on the ATA classification (Martins-Costa et al. 2016). Our metabolomics predictive model concerning M918V variant are in agreement with previous data from the literature, suggested that metabolomics may usefully contribute to the accurate functional classification of RET variants and would surely be useful for the genetic counseling of patients and their families.

\section{Limitations of the study}

We have performed the study in NIH3T3 cells, a mouse fibroblast cell line which does not express RET receptor and commonly used as cell model for functional studies and characterizations of RET mutations (ColomboBenkmann et al. 2008, Arlt et al. 2000, Borrello et al. 2011, Cosci et al. 2011, Machens et al. 2011, Prazeres et al. 2011, Colombo et al. 2015, Yang et al. 2018). However, these cells are not thyroid $C$ cells or chromaffin cells and are not a model used to explain pathophysiological mechanism.

Expression of the RET receptor was not homogeneous between the cell lines, despite the fact that all transfected cells present the same copy number of the RET gene. Other functional studies of RET variants in NIH3T3 cells presented similar inhomogeneous levels of RET protein in their various stably transfected cell lines (Borrello et al. 2011, Colombo et al. 2015). However, the morphology and growth of the seven cell lines studied here did not correlate with the protein levels since RET-L790F had more RET protein than RET-V804M or RET-C634R but had the same morphology and growth level. It cannot be excluded that, besides the intrinsic activity of RET receptors, also their levels of expression may influence signaling (p-ERK and p-AKT) and metabolic profiles. Indeed, we noted that RET-M918T, the mutant with the most discriminant metabolic profile, is also the one expressed at the highest levels.

We used here a targeted metabolomic approach using Biocrates kit, it would be valuable to test an untargeted metabolomic approach, assessing metabolomic profiles of mutant RET cells for prediction of mutant pathogenicity.

\section{Conclusion}

To our knowledge, this is the first study demonstrating the efficacy of metabolomics in testing the pathogenicity of RET mutations. This strategy could also be used for classification of controversial RET variants, such as variants of the codon 791, of modifier role of some polymorphisms, or for the evaluation of the pathogenicity of new variants discovered by high-throughput sequencing by comparing their metabolomics profiles to those of known benign or pathogenic variants.

\section{Supplementary data}

This is linked to the online version of the paper at https://doi.org/10.1530/ ERC-18-0314.

\section{Declaration of interest}

The authors declare that there is no conflict of interest that could be perceived as prejudicing the impartiality of the research reported.

\section{Funding}

The study was funded by a research grant from the University Hospital Center of Angers.

\section{Author contribution statement}

C V-D and D M-P designed and supervised the study and wrote the manuscript; C V-D and N B performed experiments; M L M performed mitochondrial function assessment; $C \mathrm{G}$ and $\mathrm{L} T$ performed metabolomics assays; G S supervised biochemical parameters assays; C V-D performed statistical analysis; FB-C, A B and D D gave technical and intellectual support; $P$ R gave intellectual support and conceptual advice for manuscript writing; J M C, C B, X D gave intellectual support. All authors offered conceptual advice and comments on the manuscript.

\section{Acknowledgements}

The authors thank Dr Massimo Santoro for kindly providing us with the RET9 plasmid. They thank the University Hospital of Angers, INSERM, CNRS and INCa for their support. They are grateful to Kanaya Malkani for revising the manuscript.

\section{References}

Arlt DH, Baur B, Wagner B \& Hoppner W 2000 A novel type of mutation in the cysteine rich domain of the RET receptor causes ligand independent activation. Oncogene 19 3445-3448. (https://doi. org/10.1038/sj.onc.1203688)

Borrello MG, Aiello A, Peissel B, Rizzetti MG, Mondellini P, Degl'Innocenti D, Catalano V, Gobbo M, Collini P, Bongarzone I, et al. 2011 Functional characterization of the MTC-associated germline RET-K666E mutation: evidence of oncogenic potential enhanced by the G691S polymorphism. Endocrine-Related Cancer 18 519-527. (https://doi.org/10.1530/ERC-10-0306)

Brunelli L, Caiola E, Marabese M, Broggini M \& Pastorelli R 2016 Comparative metabolomics profiling of isogenic KRAS wild type and mutant NSCLC cells in vitro and in vivo. Scientific Reports 628398. (https://doi.org/10.1038/srep28398)

Cheng M, Bhujwalla ZM \& Glunde K 2016 Targeting phospholipid metabolism in cancer. Frontiers in Oncology 6 266. (https://doi. org/10.3389/fonc.2016.00266)

Colombo C, Minna E, Rizzetti MG, Romeo P, Lecis D, Persani L, Mondellini P, Pierotti MA, Greco A, Fugazzola L, et al. 2015 (c) 2019 Society for Endocrinology Published by Bioscientifica Ltd. Printed in Great Britain 
The modifier role of RET-G691S polymorphism in hereditary medullary thyroid carcinoma: functional characterization and expression/penetrance studies. Orphanet Journal of Rare Diseases $\mathbf{1 0}$ 25. (https://doi.org/10.1186/s13023-015-0231-z)

Colombo-Benkmann M, Li Z, Riemann B, Hengst K, Herbst H, Keuser R, Gross U, Rondot S, Raue F, Senninger N, et al. 2008 Characterization of the RET protooncogene transmembrane domain mutation S649L associated with nonaggressive medullary thyroid carcinoma. European Journal of Endocrinology 158 811-816. (https://doi. org/10.1530/EJE-07-0817)

Cosci B, Vivaldi A, Romei C, Gemignani F, Landi S, Ciampi R, Tacito A, Molinaro E, Agate L, Bottici V, et al. 2011 In silico and in vitro analysis of rare germline allelic variants of RET oncogene associated with medullary thyroid cancer. Endocrine-Related Cancer 18 603-612. (https://doi.org/10.1530/ERC-11-0117)

Gandola YB, Perez SE, Irene PE, Sotelo AI, Miquet JG, Corradi GR, Carlucci AM \& Gonzalez L 2014 Mitogenic effects of phosphatidylcholine nanoparticles on MCF-7 breast cancer cells. BioMed Research International 2014 687037. (https://doi. org/10.1155/2014/687037)

Glunde K, Bhujwalla ZM \& Ronen SM 2011 Choline metabolism in malignant transformation. Nature Reviews Cancer 11 835-848. (https://doi.org/10.1038/nrc3162)

Hanahan D \& Weinberg RA 2011 Hallmarks of cancer: the next generation. Cell 144 646-674. (https://doi.org/10.1016/j. cell.2011.02.013)

Kaplon J, Zheng L, Meissl K, Chaneton B, Selivanov VA, Mackay G, van der Burg SH, Verdegaal EM, Cascante M, Shlomi T, et al. 2013 A key role for mitochondrial gatekeeper pyruvate dehydrogenase in oncogene-induced senescence. Nature 498 109-112. (https://doi. org/10.1038/nature12154)

Lebeault M, Pinson S, Guillaud-Bataille M, Gimenez-Roqueplo AP, Carrie A, Barbu V, Pigny P, Bezieau S, Rey JM, Delvincourt C, et al. 2017 Nationwide French Study of RET variants detected from 2003 to 2013 suggests a possible influence of polymorphisms as modifiers. Thyroid 27 1511-1522. (https://doi.org/10.1089/thy.2016.0399)

Machens A, Spitschak A, Lorenz K, Putzer BM \& Dralle H 2011 Germline RET sequence variation I852M and occult medullary thyroid cancer: harmless polymorphism or causative mutation? Clinical Endocrinology 75 801-805. (https://doi.org/10.1111/j.1365-2265.2011.04158.x)

Martins-Costa MC, Cunha LL, Lindsey SC, Camacho CP, Dotto RP, Furuzawa GK, Sousa MS, Kasamatsu TS, Kunii IS, Martins MM, et al.
2016 M918V RET mutation causes familial medullary thyroid carcinoma: study of 8 affected kindreds. Endocrine-Related Cancer $\mathbf{2 3}$ 909-920. (https://doi.org/10.1530/ERC-16-0141)

Mulligan LM 2014 RET revisited: expanding the oncogenic portfolio. Nature Reviews Cancer 14 173-186. (https://doi.org/10.1038/nrc3680)

Mulligan LM, Eng C, Attie T, Lyonnet S, Marsh DJ, Hyland VJ, Robinson BG, Frilling A, Verellen-Dumoulin C, Safar A, et al. 1994 Diverse phenotypes associated with exon 10 mutations of the RET proto-oncogene. Human Molecular Genetics 3 2163-2167. (https://doi. org $/ 10.1093 / \mathrm{hmg} / 3.12 .2163$ )

Prazeres H, Couto JP, Rodrigues F, Vinagre J, Torres J, Trovisco V, Martins TC, Sobrinho-Simoes M \& Soares P 2011 In vitro transforming potential, intracellular signaling properties, and sensitivity to a kinase inhibitor (sorafenib) of RET proto-oncogene variants Glu511Lys, Ser649Leu, and Arg886Trp. Endocrine-Related Cancer 18 401-412. (https://doi.org/10.1530/ERC-10-0258)

Reuter SE \& Evans AM 2012 Carnitine and acylcarnitines: pharmacokinetic, pharmacological and clinical aspects. Clinical Pharmacokinetics 51 553-572. (https://doi.org/10.1007/BF03261931)

Richards S, Aziz N, Bale S, Bick D, Das S, Gastier-Foster J, Grody WW, Hegde M, Lyon E, Spector E, et al. 2015 Standards and guidelines for the interpretation of sequence variants: a joint consensus recommendation of the American College of Medical Genetics and Genomics and the Association for Molecular Pathology. Genetic Medicine 17 405-424. (https://doi.org/10.1038/gim.2015.30)

Wells SA Jr, Asa SL, Dralle H, Elisei R, Evans DB, Gagel RF, Lee N, Machens A, Moley JF, Pacini F, et al. 2015 Revised American Thyroid Association guidelines for the management of medullary thyroid carcinoma. Thyroid 25 567-610. (https://doi.org/10.1089/ thy.2014.0335)

Yang J, Chen K, Zhang G, Yang QY, Li YS, Huang SZ, Wang YL, Yang W, Jiang XJ, Yan HX, et al. 2018 Structural optimization and structureactivity relationship studies of N-phenyl-7,8-dihydro-6Hpyrimido[5,4-b][1,4] oxazin-4-amine derivatives as a new class of inhibitors of RET and its drug resistance mutants. European Journal of Medicinal Chemistry 143 1148-1164. (https://doi.org/10.1016/j. ejmech.2017.09.018)

Yun J, Rago C, Cheong I, Pagliarini R, Angenendt P, Rajagopalan H, Schmidt K, Willson JK, Markowitz S, Zhou S, et al. 2009 Glucose deprivation contributes to the development of KRAS pathway mutations in tumor cells. Science 325 1555-1559. (https://doi. org/10.1126/science.1174229)

Received in final form 14 January 2019

Accepted 17 January 2019

Accepted Preprint published online 17 January 2019 (c) 2019 Society for Endocrinology Published by Bioscientifica Ltd. Printed in Great Britain 